\title{
The submillimeter properties of AE Aqr
}

\author{
Ulf Torkelsson \\ Department of Physics, University of Gothenburg, SE 41296 Gothenburg, Sweden \\ email: torkel@physics.gu.se
}

\begin{abstract}
AE Aqr is a cataclysmic variable consisting of a magnetised white dwarf with a spin period of $33 \mathrm{~s}$, and a K-dwarf companion. Because of the rapid spin the magnetosphere of the white dwarf the matter that is flowing over from the secondary cannot accrete onto the white dwarf, and rather it is ejected from the system. AE Aqr is subject to extended flaring activity at all wavelengths from radio via optical to X-rays. I present observations of AE Aqr at 345 and $850 \mathrm{GHz}$ using the LABOCA and SABOCA bolometers on APEX. These observations are good enough to distinguish individual flares at both 345 and $850 \mathrm{GHz}$, and the SABOCA observation is the first measurement of the system at $850 \mathrm{GHz}$.
\end{abstract}

Keywords. cataclysmic variables, radio emission, flares

\section{Introduction}

AE Aqr is a cataclysmic variable that consists of a K4 dwarf and a magnetic white dwarf, whose spin period is 33 (Patterson 1979), though there is no secure determination of the magnetic field strength on the white dwarf. The system has been known for a long time for its extended flaring activity (Zinner 1938). It is also both an X-ray source (Patterson et al.1980) and a radio source (Bookbinder \& Lamb 1987).

Welsh et al. (1998) found that there is no accretion disc around the white dwarf. The matter that is overflowing from the secondary is ejected by the rapidly rotating magnetosphere of the white dwarf rather than forming a disc around it. As a result of this interaction the white dwarf is spinning down at a rate of $5.6 \times 10^{-14} \mathrm{~s} \mathrm{~s}^{-1}$ (De Jager et al. (1994)). This loss of rotational energy of the white dwarf is comparable to the nonstellar luminosity of the system. The white dwarf is therefore in the so-called propeller state (Illarionov \& Sunyaev 1975). There is no other system in which we can study this mechanism in as much detail, but it can play a role in certain accreting millisecond pulsars.

Abada-Simon et al. (1993) observed AE Aqr with the Caltech Submillimeter Observatory at $240 \mathrm{GHz}$ for $1 \mathrm{~h}$. They found a mean flux of $49 \mathrm{mJy}$, but with strong fluctuations on time scales as short as 5 min. Combining these observations with other observations at lower frequencies, one finds that the mean radio emission follows an intensity $\propto \nu^{0.5}$. Furthermore they indicated that the radio emission becomes more intermittent at higher frequencies.

A scenario that has been proposed to explain the flares is that the mass transfer stream from the secondary is broken up into a stream of individual blobs (Wynn, King \& Horne 1997). When these blobs collide with the magnetosphere of the white dwarf they are heated to a high temperature by a shock wave as they are ejected from the magnetosphere. The heated blobs appear as the optical flares as they are moving out through the system. The collision between the gas stream and the magnetosphere can also accelerate electrons to relativistic velocities in the bubbles, which then produce synchrotron radiation (Kuijpers et al. 1997, Venter \& Meintjes 2006). 
Table 1. Observing log for the APEX observations AE Aqr. The first column shows the instrument that was used. The following three columns give the dates of the observations and the starting and ending times in UT. The final column gives the amount of precipitable water vapour in $\mathrm{mm}$.

\begin{tabular}{|c|c|c|c|c|}
\hline \multicolumn{2}{|c|}{ | Instrument | Date } & \multicolumn{3}{|c|}{$\mid$ Start (UT) | End (UT) | PWV (mm) | } \\
\hline LABOCA & August 2nd 2007 & $04: 48$ & $06: 16$ & $0.65-0.76$ \\
\hline LABOCA & August 3rd 2007 & $04: 34$ & $07: 58$ & $0.70-2.0$ \\
\hline LABOCA & August 4th 2007 & 03:03 & $06: 27$ & $0.63-1.2$ \\
\hline SABOCA & July 22nd 2011 & 05:05 & $06: 19$ & 0.06 \\
\hline
\end{tabular}

The rest of the paper is organised such that I present the observations in Sect. 2, and the results in Sect. 3.

\section{Observations}

I obtained observations of AE Aqr using the LABOCA and SABOCA bolometers on APEX† in 2007 and 2011, respectively (see Tab. 1). A problem with sub-mm observations is that the brightness of the sky is comparable to that of the source, and is dependent on the amount of water vapour in the atmosphere. This necessitates an observing mode in which both the brightness of the source as seen through the sky and the empty sky are measured. At the time of the LABOCA observations APEX could only work in a scanning mode, in which the telescope is continuously scanning the source and the surrounding sky. This method is inefficient for a point source such as AE Aqr, because there are gaps between the feedhorns of the bolometer, while it is an efficient way to map an extended source, because the feedhorns will then constantly be mapping different parts of the source. At the later observation with the SABOCA bolometers a wobbling mirror had been installed, and the instrument could then work in a more efficient on-off mode, in which the central bolometer cell is alternatingly measuring the source and the empty sky.

I show the light curve from one night of the LABOCA observations in Fig. 1. The other nights show similar behaviour with one or two large flares per observing session. The data have been reduced using the standard BoA $\ddagger$ reduction algorithms.

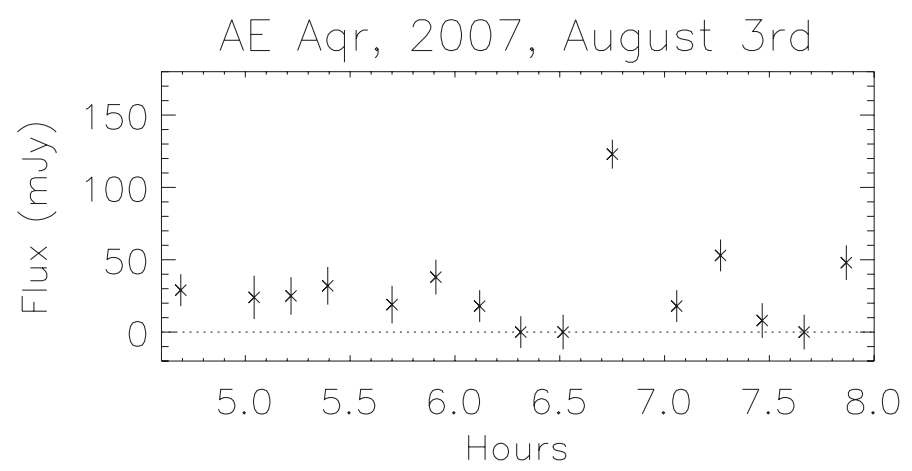

Figure 1. The light curve of AE Aqr at $345 \mathrm{GHz}$ on the 3rd of August 2007 as measured by the LABOCA bolometer on APEX. Fluxes are given in mJy and times are UT.

$\dagger$ This publication is based on data acquired with the Atacama Pathfinder Experiment (APEX). APEX is a collaboration between the Max-Planck-Institut fur Radioastronomie, the European Southern Observatory, and the Onsala Space Observatory.

$\ddagger$ http://www3.mpifr-bonn.mpg.de/div/submmtech/software/boa/boa_main.htm 


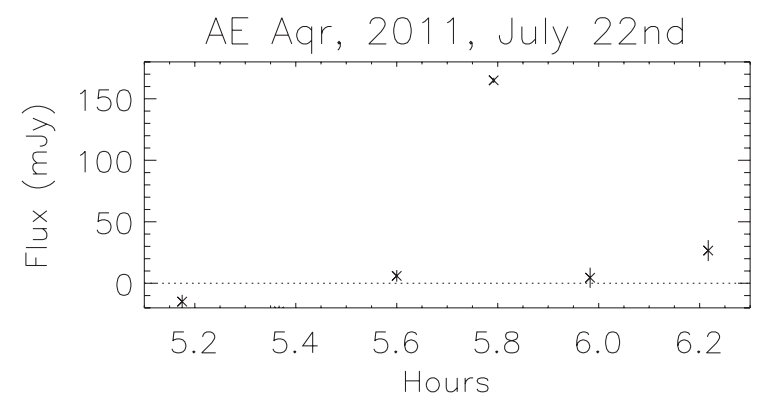

Figure 2. The light curve of AE Aqr at $850 \mathrm{GHz}$ on the 22nd of July 2011 as measured by the SABOCA bolometer on APEX. Fluxes are given in mJy and times are UT.

The SABOCA light curve is shown in Fig. 2. These data were reduced using Crush 9 . Note that the first two data points are significantly below 0. It appears that Crush is underestimating the noise in on-off observations. Anyway the SABOCA observations are of surprisingly high quality compared to the LABOCA observations. This is not only the result of that the SABOCA observations could use a more efficient observing method, but also a consequence of extremely good weather conditions with very little water vapour in the atmosphere.

\section{Results and discussion}

According to the LABOCA observations the mean radio brightness of AE Aqr in August 2007 was only 20 mJy. An extrapolation of the observations that Abada-Simon et al. (1993) carried out in 1990 suggest that the brightness should rather have been 60 mJy. The SABOCA observations from July 2011 on the other hand reach the level that is expected from an extrapolation using the $\nu^{0.5}$ spectrum. There is nothing else that indicates that AE Aqr should have been in an unusually low state during the 2007 observations, so its temporary radio faintness in August 2007 cannot be explained at present.

It is clear from the light curves that the radio emission is to a large degree intermittent. In 2007 AE Aqr was observed with LABOCA during three subsequent nights, and during each night there was one or two large flares and for the rest of the night the measured radio flux stayed within a few times the rms noise. The SABOCA observation of 2011 is even more extreme, in that AE Aqr appears to be silent outside of the one flare that was observed, but one should keep in mind that it was only observed for $1.5 \mathrm{~h}$. These data do suggest that the sub-mm emission becomes more intermittent at higher frequencies, but there is a clear need for longer time series in the SABOCA window and more sensitive observations in the LABOCA window. The latter can easily be achieved since the observations can now be done in an on-off mode.

\section{Acknowledgement}

I am grateful to the APEX staff for carrying out the observations, and for the donation funds at the University of Gothenburg for making it possible for me to participate in the IAU General Assembly.

\section{References}

Abada-Simon, M., Lecacheux, A., Bastian, T. S, Bookbinder, J. A., \& Dulk, G. A. 1993, ApJ, 406,692

I http://www.submm.caltech.edu/ sharc/crush/ 
Bookbinder, J. A. \& Lamb, D. Q. 1987, ApJ, 323, L131

de Jager, O. C., Meintjes, P. J., O'Donoghue, D., \& Robinson, E. L. 1994, MNRAS, 267, 577

Illarionov, A. F. \& Sunyaev, R. A. 1975, $A \mathscr{E} A, 39,185$

Kuijpers, J., et al. 1997, A\&̈A, 322, 242

Patterson, J. 1979, ApJ, 234, 978

Patterson, J., Branch, D., Chincarini, G., \& Robinson, E. L. 1980, ApJ, 240, L133

Venter, L. A. \& Meintjes, P. J. 2006, MNRAS, 366, 557

Welsh, W. F., Horne, K., \& Gomer, R. 1998, MNRAS, 298, 285

Wynn, G. A., King, A. R., \& Horne, K. 1997, MNRAS, 286, 436

Zinner, E. 1938, Astr. Nach., 265, 345 